\title{
Phase transformation and microstructures in Ni-Mn-Ga ferromagnetic shape memory alloys
}

\author{
K. Tsuchiya, H. Nakamura, D. Ohtoyo, H. Nakayama, M. Umemoto \\ and $\mathrm{H}$. Ohtsuka ${ }^{1}$
}

\author{
Department of Production Systems Engineering, Toyohashi University of Technology, \\ Toyohashi, Aichi 441-8580, Japan \\ ${ }^{1}$ National Research Institute of Metals, Tsukuba, Ibaraki 305-0003, Japan
}

\begin{abstract}
Composition dependence of martensitic transformation and magnetic transformation has been studied on $\mathrm{Ni}-\mathrm{Mn}-\mathrm{Ga}$ ferromagnetic shape memory alloys. A good correlation was found between the transformation temperatures and valence electron density (e/a). Martensitic transformation temperature increases rapidly with $\mathrm{e} / \mathrm{a}$, while the Curie temperature exhibits only a mild dependence. Crystal structure of martensite also changes with e/a. At a lower e/a (near the stoichiometric Heusler composition) the martensite was bct with the modulation of five layers period of $\{110\}_{\mathrm{L} 21}$ planes; at a higher e/a, it was fct without the modulation. Transformations from the parent phase to an intermediate phase and then to martensite phase were observed in situ using a cold stage for Ni-23.0Mn-25.6Ga. The sample exhibits so-called "tweed" structures in the parent phase. The tweed contrast becomes more pronounced as the temperature approaches $M_{s}$. This is accompanied by increasing intensity of diffuse streaks along $<110\rangle$ directions. At $230 \mathrm{~K}$ the satellite spots appeared at $1 / 6<220>$ positions, corresponding to the intermediate phase. On further cooling martensite plate formed and the structure changed continuously to the modulated bct.
\end{abstract}

\section{INTRODUCTION}

Martensitic transformation in $\mathrm{Ni}_{2} \mathrm{MnGa}$ alloy was discovered by Webster $e$ t al. [1]. They have discovered an apparent decrease of low field magnetization at martensitic transformation temperature. This is due to low crystallographic symmetry of martensite phase and, as a result, a high magnetocrystalline anisotropy. Since then, there have been a number of studies regarding various aspects of phase transformations in this alloy. Furthermore, Ullakko et al. [2] have demonstrated that martensite phase in Ni-Mn-Ga alloy exhibits anomalous magnetostriction of $0.15 \%$; this value is comparable to so-called giant magnetostriction in $\mathrm{Tb}_{0.3} \mathrm{Dy}_{0.7} \mathrm{Fe}_{2}$ (commercially available as Terfenol-D). The observed large magnetostriction is due to the magnetically induced twinning/detwinning in the martensite and thus appropriated to call it as "twinning magnetostriction". Temperature dependnece of magnetostriction in Ni-Mn-Ga alloy has been studied by the present authors [3]. James et al. have shown that one-way strain of up to $5 \%$ can be magnetically induced in a single crystal of martensite phase [4]. Thus this alloys is a good candidate for a new magnetic actuator materials and is attracting much attention [5-7].

$\mathrm{Ni}-\mathrm{Mn}-\mathrm{Ga}$ alloy also provides excellent opportunity to gain a physical insight regarding fundamental issues on phase transformation, such as, magneto-elastic interaction and premartensitic phenomena. Existence of intermediate phase transformation that precedes martensitic transformation was suggested by various experiments such as inelastic neutron scattering [8], elastic modulus and internal friction measurements [9], calorimetric measurements and ac magnetic susceptibility measurements [10].

Present study was undertaken to clarify the composition dependence of phase transformation behavior and crystal structures of martensite in $\mathrm{Ni}-\mathrm{Mn}-\mathrm{Ga}$ ferromagnetic shape memory alloys. 


\section{EXPERIMENTAL}

Alloy ingots with various compositions were prepared by a tri-arc melting furnace under an argon atmosphere. The ingots were homogenized at $1073 \mathrm{~K}$ for $48-72$ hours in an argon atmosphere. They were then cut for various measurements and analysis. Each sample was given a final heat treatment of 1 hour at $1073 \mathrm{~K}$ in an argon atmosphere, followed by quenching into water. Martensitic transformation temperature was measured using Rigaku DSC $8230 \mathrm{~L}$ at a heating/cooling rate of $10 \mathrm{~K} / \mathrm{min}$. Magnetic field-magnetization curves were obtained by RIKEN BHV-55 vibrating sample magnetometer (VSM) with an applied field up to $15 \mathrm{kOe}\left(1.19 \mathrm{MA} \mathrm{m}^{-1}\right)$ at room temperature. SQUID measurements were done on QUANTUM MPMS-7. For TEM observations a $\phi 3 \mathrm{~mm}$ disc was electropolished using nitric acid/methanol solution at $253 \mathrm{~K}$. The sample was observed on JEOL-200CX or JEOL-2010 using sample cooling stage.

\section{RESULTS}

\subsection{Transformation temperatures}

Typical DSC profiles observed in $\mathrm{Ni}-\mathrm{Mn}-\mathrm{Ga}$ alloys are shown in Fig. 1 (a) - (c) for Ni-23.0Mn-25.6Ga (e/a = 7.52), Ni-23.1-24.6Ga $(\mathrm{e} / \mathrm{a}=7.59)$ and $\mathrm{Ni}-20.0 \mathrm{Mn}-25.0 \mathrm{Ga}(\mathrm{e} / \mathrm{a}=7.65)$ alloys, respectively. In Fig. 1(a), step-like features in the DSC profiles denoted by the arrows correspond to ferromagnetic transformation and can be taken here as the Curie temperature $\left(T_{c}\right)$. Hysteresis for martensitic and magnetic transformation temperatures is about $15 \mathrm{~K}$ and $5 \mathrm{~K}$, respectively. In Fig. 1(b) minor peaks denoted by triangles can be recognized in addition to the martensitic transformation peaks and magnetic transformation peaks. The origin of the minor peaks may be an intermediate phase, previously reported for near stoichiometric alloys. It was reported that for an alloy with near Heusler composition the latent heat for the intermediate phase transformation is very small and detectable only when a large amount of sample was tested [11]. The present results suggest that the latent heat becomes large enough to be

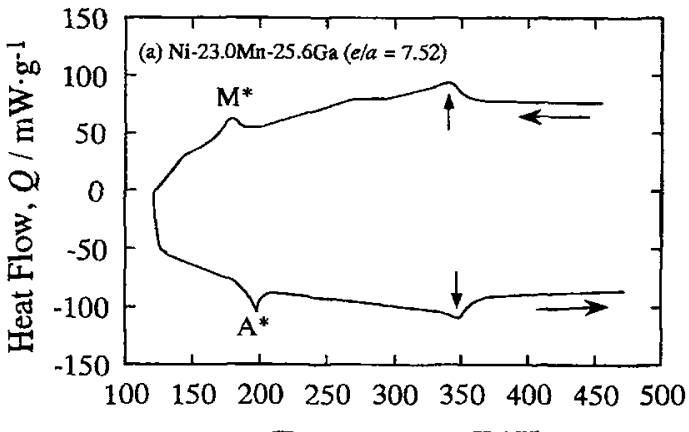

Temperature, $T / \mathrm{K}$
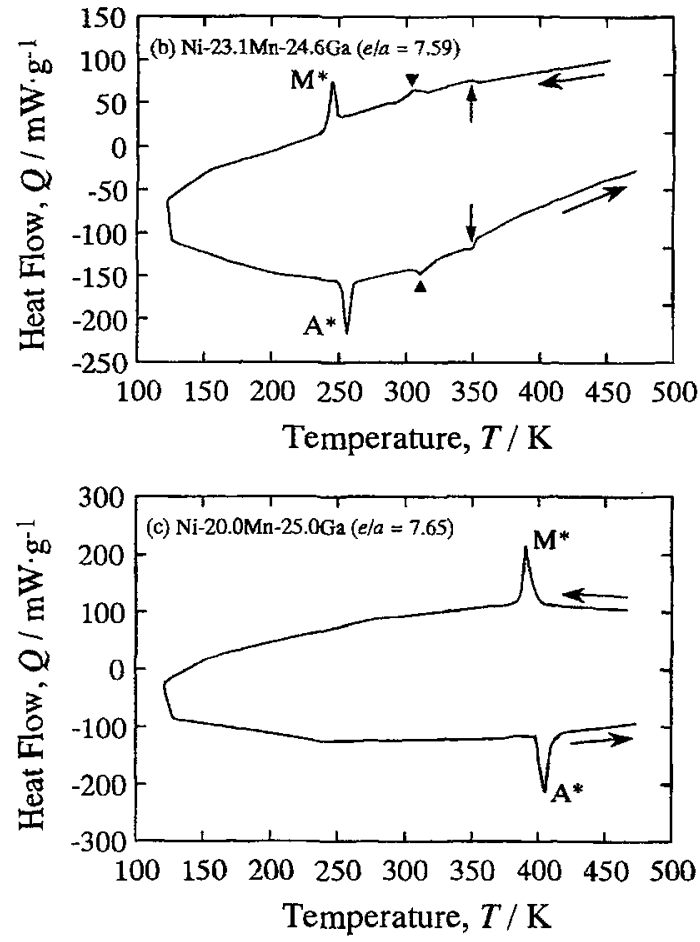

Figure 1: Typical DSC curves for Ni-Mn-Ga alloys. 
detected in conventional DSC measurements in off-stoichiometric alloys. Figure 1 (c) is a DSC profile for $\mathrm{Ni}-20.0 \mathrm{Mn}-25.0 \mathrm{Ga}$ alloy associated with a higher martensitic transformation temperature. In this alloy only the peaks corresponding to martensitic transformation can be seen.

Peak temperatures corresponding to forward martensitic transformation, $M^{*}$, are plotted as a function of valence electron concentration, e/a, in Fig. 2. The single crystal data by Webster et al. are also indicated for the stoichiometric Heusler composition corresponding to e/a of 7.5. $\mathrm{M}^{*}$ increases monotonically with e/a. This suggests that electron concentration is a pertinent parameter to determine the stability of the parent phase and martensite phase as in the case of $\mathrm{Cu}-\mathrm{Zn}-\mathrm{Al}$ shape memory alloys. The Curie temperatures as well as temperatures corresponding to the minor peaks in DSC profiles, $T_{I}$, are also plotted in Fig. 2. While the $M^{*}$ depends on e/a rather strongly, the e/a dependence of $T_{c}$ and $T_{I}$ is less significant.

Temperature dependence of magnetization under $1 \mathrm{kOe}$ was determined by SQUID measurement and is shown in Fig. 3 for Ni-23.0Mn-25.6Ga. The Curie temperature, $M_{s}$ and $\mathrm{M}_{\mathrm{f}}$ can be determined to be $363 \mathrm{~K}, 189 \mathrm{~K}$ and $178 \mathrm{~K}$, respectively. In addition to the large changes in the magnetization at the Curie temperature, $M_{s}$ and $M_{f}$, a small dip exists at around $230 \mathrm{~K}$ as indicated in the inset of Fig. 3. This dip was not observed in a measurement under a higher magnetic field of $10 \mathrm{kOe}$.

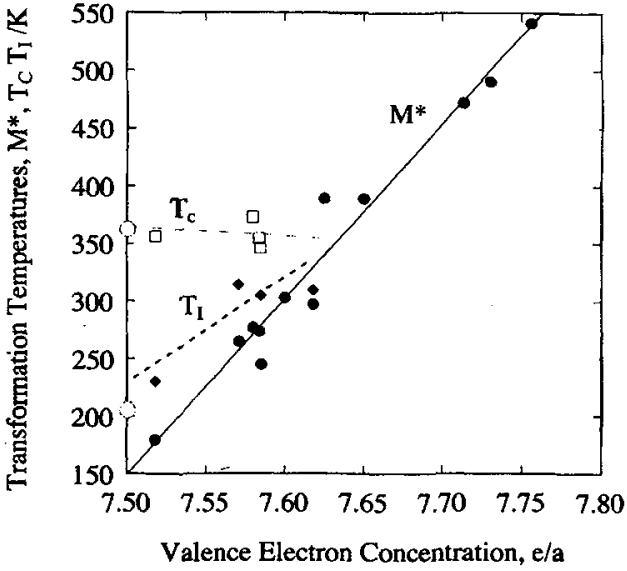

Figure 2: Transformation temperatures vs. e/a. $\mathbf{M}^{*}$ $(\mathrm{C}), \mathrm{T}_{\mathrm{C}}(\square)$ and $\mathrm{T}_{\mathrm{I}}(\diamond)$.

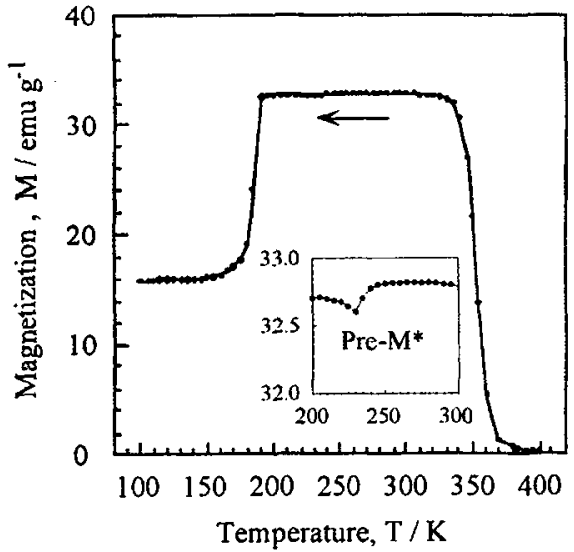

Figure 3: Magnetization as a function of temperature. Applied field $=1 \mathrm{kOe}$.

\subsection{TEM observations}

Figs. 4 (a) and (b) are a bright field (BF) TEM image and selected area diffraction (SAD) for $\mathrm{N}-16.7 \mathrm{Mn}-24.9 \mathrm{Ga}(\mathrm{e} / \mathrm{a}=7.76)$ alloy obtained at room temperature, respectively. It was revealed by the inspection of the SAD (Fig. 4(b)) and dark field analysis that the striations in the martensite plates are internal twins. It can be also seen that the boundaries between the martensite plates are serrated and exhibit strong strain contrast. Observation at a higher magnification revealed that the strong strain contrast was due to the crossing of internal twins. The SAD (Fig. 4(b)) indicates that the structure of martensite is twinned fct with $\mathrm{c} / \mathrm{a}=0.8735$ (here the ordered structure is neglected and the parent phase was regarded as bcc). If we 

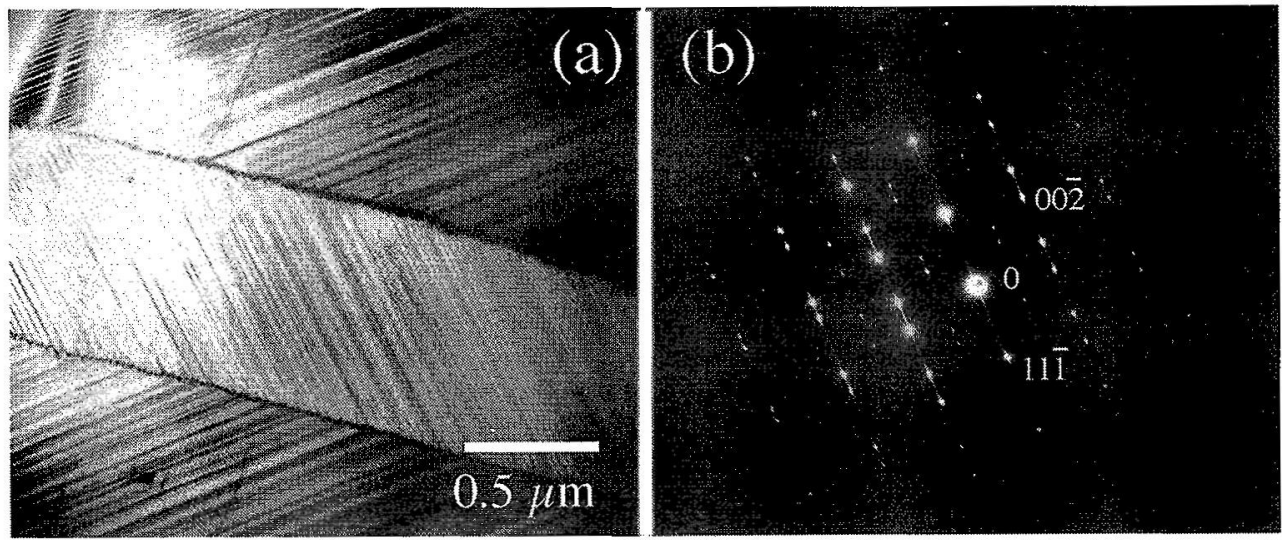

Figure 4: (a) TEM bright filed image and (b) selected area diffraction of Ni-16.7Mn-24.9Ga alloy.

refer to the original $\mathrm{L} 2_{1}$ lattice this corresponds to an extension along one of the $\langle 100\rangle_{\mathrm{L} 2 \mathrm{I}}$ axis to $(\mathrm{c} / \mathrm{a})_{\mathrm{L} 21}=1.23$.

Martensitic transformation in Ni-23.0Mn-25.6Ga alloy was observed in-situ by TEM using a cold stage. Fig .5 shows a series of SAD patterns with an electron beam direction parallel to $[111]_{\mathrm{L} 21}$. At room temperature (Fig. 5(a)), very diffuse streaks along $\langle 110\rangle$ direction can be seen. The streaks become more pronounced and sharper with decreasing temperature (Fig. 5(b)). It was verified by tilting the sample that the streaks in radial direction are due to double diffraction. At about $230 \mathrm{~K}$, satellite spots emerged at the ends of the streaks (Fig. 5(c)). The distance between the satellite spots and main $<220>$ spots is one sixth of the $\left|\mathrm{g}_{220}\right|$. This temperature corresponds to the small dip on the magnetizationtemperature curve (Fig. 3). By further decreasing the temperature, the satellite spots on $\langle 1-10\rangle$ streaks increased their intensity at the expense of those on $\langle 10-1\rangle$ and $\langle 0-11\rangle$ streaks. At $183 \mathrm{~K}$ (Fig. 5(d)), which is near $\mathrm{M}_{\mathrm{f}}$ temperature, new extra spots appeared between two satellites along the $\langle 1-10\rangle$ directions and martensite plates started to form. At this temperature the extra spots are in incommensurate positions with respect to the main spots. The intensity of extra spots increases by further cooling to $123 \mathrm{~K}$ (Fig. 5(e)). At this temperature the extra spots are on $1 / 5<220>$ positions. The basic structure is bct with $(\mathrm{c} / \mathrm{a})_{\mathrm{bcc}}=0.94$.

\section{DISCUSSION}

Observed changes in the SAD patterns on cooling seem to be consistent with the temperature dependence of phonon dispersion characteristics observed by Zheludev et al. [8]. They have found a pronounced softening of $1 / 3<110>$ phonon in $\mathrm{TA}_{2}$ branch. Similar phonon softening was also reported for $\mathrm{Ni}-\mathrm{Al}$ [12], and Ti-Ni-Fe alloy [13]. There exists, however, a significant difference between these three systems. In the case of $\mathrm{Ni}-\mathrm{Al}$, the softening is incomplete but it persists to just above $\mathrm{M}_{\mathrm{s}}$ temperature. In $\mathrm{Ti}-\mathrm{Ni}-\mathrm{Fe}$ alloy the softening is complete and it directly leads to a transformation to $\mathrm{R}$ phase. In Ni-Mn-Ga the softening is incomplete and the corresponding phonon energy is the lowest at the temperature $40 \mathrm{~K}$ above $\mathrm{M}_{s}$ and it increases with further increasing temperature. This peculiar behavior is also apparent in the temperature dependence of elastic modulus, $\mathrm{C}^{\prime}\left(=\left(\mathrm{C}_{11}-\mathrm{C}_{12}\right) / 2\right)[10]$.

In the present study a small dip was found in the magnetization $v s$. temperature curve at $230 \mathrm{~K}$, which is about $40 \mathrm{~K}$ above $\mathrm{M}_{\mathrm{s}}$. This dip temperature coincides with the temperature at which the satellite spots emerge at the $1 / 6<220>$, suggesting that the modulated structure 

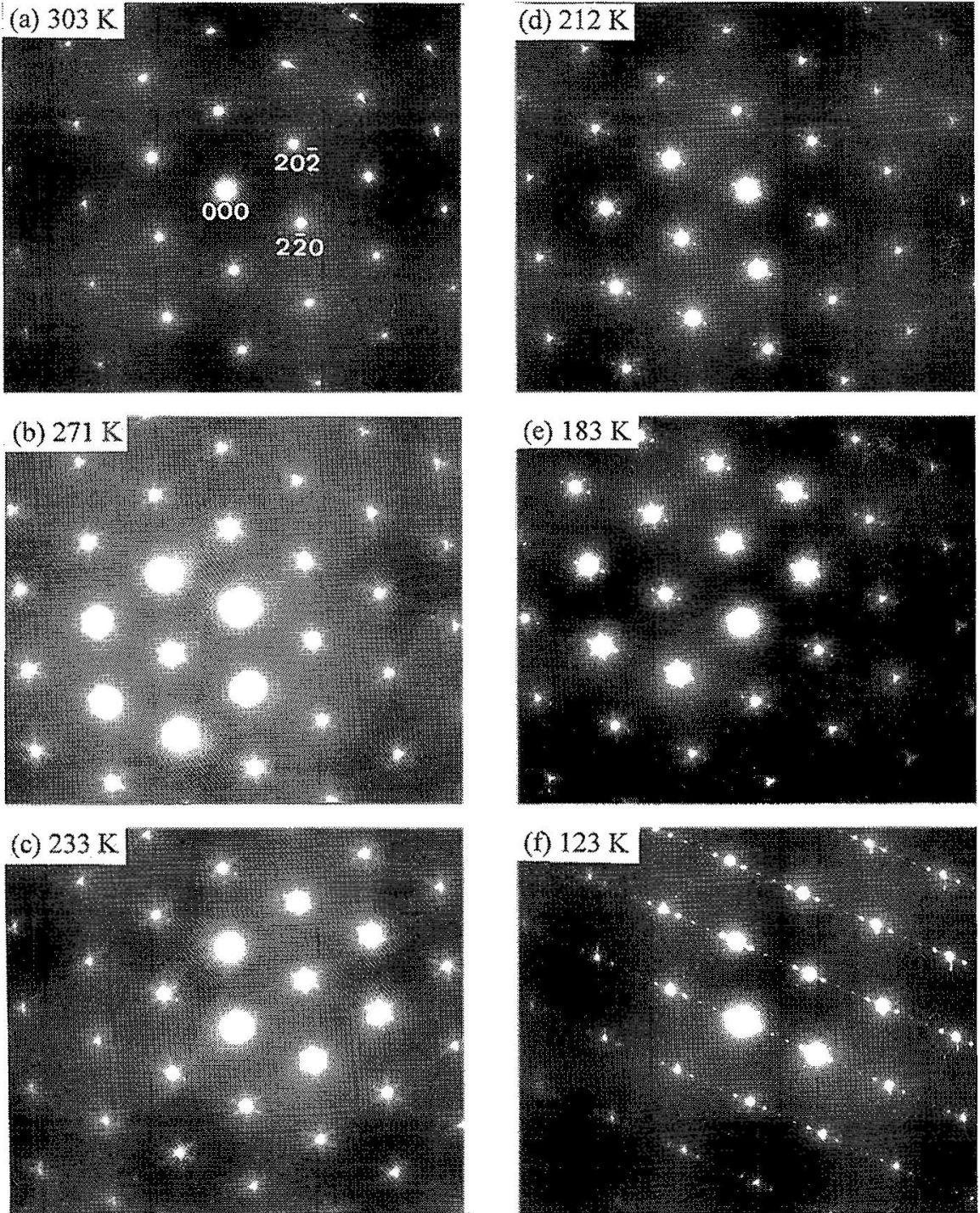

Figure 5: A series of selected area diffraction at various temperatures for $\mathrm{Ni}-23.0 \mathrm{Mn}-25.6 \mathrm{Ga}$ alloy.

with the period of 6 layers of $\{110\}_{\mathrm{L} 2 \mathrm{I}}$ planes.

Although no significant change in microstructure accompanies the intermediate phase transformation, martensitic transformation is accompanied by the formation of martensite plates. The satellite spots at $1 / 6<220>$ positions remain, but on further cooling, their position shifted away from the main spots to $1 / 5<220>$ positions accompanied by a gradual increase in the extra spots. The extra spots were in incommensurate positions at 183 $\mathrm{K}$; when cooled to $123 \mathrm{~K}$ they were at commensurate positions. This suggests that the transformation from the intermediate phase to martensite is continuous.

Crystal structure of martensite is also dependent on composition. In alloy with near stoichiometric Heusler composition $(\mathrm{e} / \mathrm{a}=7.5)$ it is bet which forms as a result of the Bain 
contraction along one of $\langle 100\rangle_{\mathrm{L} 21}$ directions. Meanwhile it is fct associated with the Bain extension in alloys with higher e/a. Our preliminary investigations suggested that the phase boundary between the bct and fct is at e/a of around 7.6. It should be noted that this is where the martensitic transformation temperature line and Tc line cross each other (Fig. 1). Recent neutron diffraction study by Brown et al. [14] concluded that the transformation to bet phase is driven by the band Jahn-Teller effect. Electronic band structure calculation by Ishida et al.[15] also supports this result. The calculation also indicates that the band Jahn-Teller effect is effective when the parent phase is ferromagnetic. Thus for the transformation at higher e/a where martensite forms from paramagnetic parent phase, the driving force may have a different origin, leading to the different crystal structures of martensite.

\section{CONCLUSIONS}

The present investigation has shown that the e/a is an effective parameter to rationalize the composition dependence of transformation temperatures and martensite crystal structure in $\mathrm{Ni}-\mathrm{Mn}-\mathrm{Ga}$ alloy. In situ observations of intermediate and martensitic transformations were also made in TEM with a cold stage. The transformation from intermediate phase to martensite phase was found to be continuous.

\section{References}

[1] Webster P. J., Ziebeck K. R. A., Town S. L. and Peak M. S., Philos. Mag., 49B (1984) 295-310.

[2] Ullakko K., Huang J. K., Kokorin V. V. and Handley R. C. O., Scrip. Metall., 36 (1997) 1133-1138.

[3] Tsuchiya K., Ohashi A., Ohtoyo D., Nakayama H., Umemoto M. and McCormick P. G., Trans. Jpn. Inst. Metals., 41 (2000) to be published.

[4] James R. D., Tickles R. and Wuttig M., Mater. Sci. Eng., A273-275 (1999) 320-325.

[5] Chernenko V. A., Cesari E. and Kokorin V. V., Scrip. Metall. Mater, 33 (1995) 1239-1244.

[6] Chernenko V. A., Scripta Mater, 40 (1999) 523-527.

[7] Vasil'ev A. N., Bozhko A. D., Khovailo V. V., Dikshtein I. E., Shavrov V. G., Buchelnikov V. D., Matsumoto M., Suzuki S., Takagi T. and Tani J., Phys. Rev., 59 (1999) 1113-1120.

[8] Zheludev A., Shapiro S. M. and Wochner P., Phys. Rev, 51 (1995) 11310-11314.

[9] Cesari E., Chernenko V. A., Kokorin V. V., Pons J. and Segui C., Acta Mater., 45 (1997) 999-1004.

[10] Manosa L., Gonzalez-Comas A., Obrado E. and Planes A., Phys. Rev., 55 (1997) 11068-11071.

[11] Planes A., Obrado E., Gonzalez-Comas A. and Manosa L., Phys. Rev. Lett., 79 (1997) 3926-3929.

[12] Shapiro S. M., Yang B. X., Noda Y., Tanner L. E. and Schryvers D., Physical Rev. B., 44 (1991) 9301-9313.

[13] Zhao G. L. and Harmon B. N., Physical Rev. B., 45 (1992) 2818-2824.

[14] Brown P. J., Bargawi A. Y., Crangle J., Neumann K.-U. and Ziebeck K. R. A., J. Phys. : Condens. Matter, 11 (1999) 4715-4722.

[15] Ishida S., Sugimura S., Fujii S. and Asano S., Trans. Mater. Res. Soc. Japan., 25 (2000) 513-516. 